ARTÍCULOS

\title{
Estrategias de evaluación formativa y desempeño docente en la evaluación para el aprendizaje Educación Básica
}

\section{Regular}

\author{
Formative assessment strategies and teacher \\ performance in the assessment for learning in Regular \\ Basic Education
}

Dolorier Zapata, Rosa Guillermina

\author{
Rosa Guillermina Dolorier Zapata \\ Universidad Nacional de Educación Enrique Guzmán y Valle, \\ Perú \\ rdolorierzapata@hotmail.com
}

Roxana Marlene Villa López

Universidad Nacional de Educación Enrique Guzmán y Valle, Perú

rvilla@une.edu.pe

Aristida Rufina Cisneros Flores

Universidad Nacional de Educación Enrique Guzmán y Valle, Perú

rufinacisneros31@gmail.com

\section{Delectus}

Instituto Nacional de Investigación y Capacitación Continua, Perú ISSN-e: 2663-1148

Periodicidad: Semestral

núm. 2, 2021

publicaciones.iniccperu@gmail.com

Recepción: 24 Mayo 2021

Aprobación: 17 Junio 2021

Publicación: 01 Julio 2021

Esta licencia permite a otros entremezclar, ajustar y construir a partir de su obra con fines no comerciales, y aunque en sus nuevas creaciones deban reconocerle su autoría y no puedan ser utilizadas de manera comercial, no tienen que estar bajo una licencia con los mismos términos.

\section{(i) (3)}

Esta obra está bajo una Licencia Creative Commons AtribuciónNoComercial 4.0 Internacional.
Resumen: El objetivo de la presente investigación fue determinar de qué manera se relacionan la argumentación teórica de las estrategias de evaluación formativa y el desempeño de la evaluación para el aprendizaje de los docentes de Educación Básica Regular (EBR) que conducen la práctica preprofesional de la Facultad de Educación Inicial de la Universidad Nacional de Educación Enrique Guzmán y Valle, Perú (UNE). Es un estudio cuantitativo, de tipo descriptivo-correlacional. Se trabajó con el diseño no experimental transversal correlacional, la población estuvo conformada por los 15 docentes de Práctica Preprofesional, siendo esta una muestra probabilística. Para la medición de las variables, se utilizó el cuestionario. Los resultados descriptivos indicaron que el $46,7 \%$ (7) realizan un argumento teórico de las estrategias de la evaluación formativa regular; asimismo, de los 15 encuestados se puede observar que el 66,7 \% (10) muestran un desempeño docente en la evaluación para el aprendizaje en EBR satisfactorio. Concluyéndose que la argumentación teórica de las estrategias de evaluación formativa se relaciona con el desempeño de la evaluación para el aprendizaje de los docentes de EBR que conducen la práctica preprofesional $(\mathrm{p}<0,05$, Rho de Spearman $=0,804$; correlación positiva alta).

Palabras clave: argumentación teórica, estrategias de evaluación, evaluación formativa, desempeño docente, evaluación para el aprendizaje.

Abstract: The objective of this research was to determine how the theoretical argumentation of formative assessment strategies and the performance of assessment for learning of teachers of Regular Basic Education (RBE) who conduct the pre-professional practice of the Faculty of Initial Education of the Enrique Guzmán y Valle National University of Education, Peru (NUE) are related. This is a quantitative, descriptive- correlational study. It was worked with a non-experimental cross-sectional correlational design, the population consisted of the 15 teachers of Preprofessional Practice, being this a probabilistic sample. The questionnaire was used to measure the variables. The descriptive results indicated that $46.7 \%$ (7) make a theoretical argument of the estrategies of regular formative assessment; likewise, of the 15 respondents it can be observed that $66.7 \%$ (10) show a satisfactory teaching performance in assessment for learning in RBE. Concluding that the theoretical argumentation of formative assessment strategies is related to the performance of assessment for learning of RBE teachers 
conducting pre-professional practice $(\mathrm{p}<0.05$, Spearman's Rho $=0.804$; high positive correlation).

Keywords: theoretical argumentation, assessment strategies, formative assessment, teacher performance, assessment for learning.

\section{INTRODUCCIÓN}

Las diversas pruebas nacionales e internacionales en las que Perú ha participado proporcionan una situación poco alentadora. Más de la mitad de los estudiantes en promedio (distintas áreas evaluadas), no logran las competencias básicas. Lo paradójico está en que el porcentaje de desaprobados en las escuelas está por debajo del 10\% a nivel nacional. Esta situación crea un contexto desalentador: los estudiantes aprueban, pero no aprenden.

Dicha situación no es ajena a la universidad o instituciones donde se ha heredado las mismas prácticas evaluadoras en sus aulas. Pero la situación se agrava, si la universidad en cuestión es formadora de profesionales en educación, en la cual ya no es solo una situación diagnóstica que se debe solucionar en el tiempo; es por el contrario, una urgencia que necesita ser corregida en el menor tiempo posible, pues está en juego la educación de un país.

Para mediar este proceso, la educación básica ha incorporado una nueva forma de evaluar (Ministerio de Educación [Minedu], 2017), la cual se denomina evaluación formativa, como respuesta a las tendencias pedagógicas contemporáneas y la necesidad de trasladar la concepción de evaluación a una evaluación para el aprendizaje. Este cambio coincide con la legitimación de la política educativa en torno a las competencias.

En tal sentido, pasar de evaluar lo incorrecto y lo correcto (aprobados y desaprobados), a formas más complejas (estándares, desempeños, evidencias, actuaciones) significa un cambio conceptual de gran envergadura, en la medida que la práctica evaluadora arraigada se sostiene en lo que mejor resulta para el docente y, generalmente, esta ha sido delimitada por su propia experiencia de evaluación. Lo anterior genera un pasivo significativo para cualquier cambio que se quiera hacer en la gestión de la evaluación del docente en el aula.

A inicios del 2017, se implementó en todas las escuelas del país la evaluación formativa, y sobre este enfoque fueron evaluadas las docentes de educación inicial. Solo en el primer tramo de evaluación, desaprobó el 1\%; esto significa que tanto el enfoque como la aplicación de la misma es de amplio dominio. Esta situación obligó a identificar cuáles son los argumentos teóricos que poseen las docentes sobre la evaluación formativa y su posterior coherencia con la aplicación de la misma en los espacios educativos reales. Uno de los espacios donde se adquiere los comportamientos evaluadores es en el tránsito de convertirse en docente, lo cual se ubica en las prácticas preprofesional, que tiene una duración de dos años y medio, en la que el estudiante interviene pedagógicamente en aulas escolares según el nivel educativo de su especialidad.

Actualmente, el Estado peruano invierte millones para implementar capacitaciones, acompañamientos, mentoría, entre otras estrategias para hacer que la evaluación formativa se aplique en los contextos educativos. Pero parece que aún priman conductas ancestrales en la evaluación, que buscan aprobados, pero no el aprendizaje, y hacen que las estrategias válidas en la evaluación formativa, como la autoevaluación se conviertan en una estrategia que facilita una buena calificación y no la reflexión deseada, situación que quizás se reproduzca en las instituciones formadoras de la Universidad Nacional de Educación Enrique Guzmán y Valle, Perú (UNE).

La evaluación formativa es aquella evaluación que tiene lugar en el momento de enseñar y aprender, realizada por el docente o los propios estudiantes, para tomar decisiones oportunas, respondiendo de manera eficiente a los datos y evidencias recogidas, que contienen la necesidad o situación actual del estudiante, permitiendo al docente introducir cambios para saciar dicha necesidad cognitiva.

Por otro lado, la evaluación, para ser formativa, no basta que sea continua y permanente, necesita tener una intención de levantar un diagnóstico para mejorar la actuación del estudiante en favor de su aprendizaje, según lo señala Perrenoud (1999), “... una evaluación es formativa si, al menos en la mente del profesor, está destinada a contribuir a la regulación de los aprendizajes en curso” (p. 54). Si solo se levanta información 
como una mera mecanización de procesos didácticos y la intención es calcular los puntajes obtenidos, más que mejorar la actuación realizada, la evaluación formativa pierde su naturaleza.

Existen diversos puntos de vista sobre las estrategias que debe usar la evaluación formativa para hacer más efectiva su tarea, una de ellas la proponen Allal \& Perrenoudl (1980), quienes la dividen en cuatro situaciones debidamente delimitadas: 1. recolección de información primordial que se circunscribe en recopilar datos sobre lo que sabe y las representaciones que tiene el estudiante sobre la actividad que realiza, 2. observación del estudiante por parte del docente, cuando realiza la tarea, 3. registro de lo observado en instrumentos debidamente validados para delimitar los pasos realizados en la resolución de la tarea, y 4. adaptación de estrategias para atender las necesidades educativas que presenta el estudiante y pueda cumplir con éxito la tarea.

Con respecto a la evaluación para el aprendizaje, Assessment Reform Group fue la institución que acuño el término de evaluación para el aprendizaje, como el proceso que crea las mejores condiciones para que se produzca el aprendizaje. La evaluación para el aprendizaje tiene como sustento el marco de la evaluación formativa, entendida como la comparación entre los niveles reales y los de referencia, que permite identificar la brecha que luego es modificada para alcanzar la meta deseada (Sadler, citados en Shepard, 2006); (Black \& Dilan, 1998). Este tipo de evaluación permite valorar el progreso del estudiante de forma frecuente (OCDE,

2005), levantando información para que el docente pueda ajustar las sesiones, unidades y programas, en favor de la demanda educativa y no necesariamente solo para evitar o corregir los errores hallados en la construcción del aprendizaje.

Relacionado con el desempeño efectivo del docente en el enfoque de la evaluación formativa en el aula, según la investigación realizada por Ravela et al. (2004) donde se recoge información sobre las experiencias en evaluación formativa (evaluación para el aprendizaje) de los profesores en Uruguay, Colombia, Perú y Chile. Para el caso de Perú, se encontraron algunas características, a partir de una entrevista con docentes quienes señalaron: no hacer ningún tipo de devolución al entregar las tareas corregidas, las devoluciones siempre las dan en positivo, priman como forma de retroalimentación los elogios o aplausos, y piensan que la evaluación formativa está ligado a la formación integral del estudiante (mejora de la disciplina, puntualidad, entre otros); sobre este contexto se desarrolla los desempeños del docente peruano.

La retroalimentación no es solo una pregunta o un procedimiento para levantar un diagnóstico del recuerdo estudiantil, sino que también es una estrategia que involucra tres preguntas básicas que permiten que los estudiantes puedan mejorar su desempeño. Estas son: ¿hacia dónde vamos?, ¿dónde estamos? y ¿cómo podemos seguir avanzando? Estas preguntas hacen que se vuelva poderosa la retroalimentación en vista que: (a) saben en qué consiste el éxito que persiguen, (b) son conscientes de que pretendemos reducir la brecha que existe entre el punto en el que se encuentra en el que se necesita alcanzar, y (c) entienden que lo más importante en que les demos información sobre el punto al que tienen que dirigirse (Hattie \& Yates, 2018, p.87).

La gestión de la evaluación formativa (conocimiento y aplicación) debe ser analizada con fines de mejora, reconocer qué tipo de conceptos o fundamentos teóricos son los que disponen los docentes de la práctica preprofesional de la UNE, específicamente de la Facultad de Educación Inicial. Lo que ellos imparten a sus estudiantes es prioridad institucional, en favor de asegurar la formación profesional de lo que requiere la nación.

En ese sentido, se formula el problema de la manera siguiente, ¿de qué manera la argumentación teórica de las estrategias de la evaluación formativa se relaciona con el desempeño de la evaluación para el aprendizaje de los docentes de Educación Básica Regular (EBR) que conducen la práctica preprofesional de la Facultad de Educación Inicial de la UNE? El objetivo de esta investigación fue determinar de qué manera se relacionan la argumentación teórica de las estrategias de evaluación formativa y el desempeño de la evaluación para el aprendizaje de los docentes de EBR que conducen la práctica preprofesional de la Facultad de Educación Inicial de la UNE.

Como hipótesis se plantea que la argumentación teórica de las estrategias de evaluación formativa se relaciona con el desempeño de la evaluación para el aprendizaje de los docentes de la práctica preprofesional 
de la Facultad de Inicial de la Universidad Nacional de Educación.

\section{METODOLOGÍA}

Se empleó el enfoque cuantitativo, en vista de que se medirán las variables a partir de los indicadores expuestos de manera explícita en cada una de las dimensiones que la investigación ha priorizado. El tipo de investigación es descriptiva correlacional, busca identificar qué tipo de relación existe entre las dos variables. Asimismo, se empleó el método descriptivo, ya que solo busca describir la relación existente entre las dos variables, a partir del análisis e interpretación sistemática del comportamiento de sus dimensiones en un tiempo específico.

Es un estudio correlacional, no causal, ya que solo busca identificar el tipo de relación que presentan las variables dentro del contexto específico del estudio. Se utilizó el método hipotético-deductivo, este procedimiento parte de afirmaciones que son denominadas hipótesis y se busca demostrarlas para luego deducir conclusiones que pueden ser confrontadas con los hechos de la problemática. Se utilizó también el método descriptivo, este permite conocer, analizar, describir y sobre todo interpretar de forma ordenada todo un conjunto de elementos o sucesos correspondientes a una variable de estudio. Así también, el método analítico-sintético parte de un análisis empírico y sus aspectos esenciales fueron operacionalizados en categorías teniendo un alto nivel de generalización, caracterización y abstracción. Por último, se utilizó el método inductivo-deductivo; de acuerdo a este, se inició la investigación en función a hechos concretos de donde se realizó la problematización, categorización y conceptualización de las variables.

La población estudio estuvo conformada por los 15 docentes de práctica preprofesional de la Facultad de Inicial de la UNE, en el semestre 2020-I. La muestra fue conformada con los 15 docentes que conducen la práctica preprofesional del semestre 2020-I. En este caso, es una muestra censal, porque se ha seleccionado el 100\% de la población, al ser considerado un número manejable para la investigación. La técnica fue la encuesta. Según Hernández et al. (2014), entre las diversas técnicas de recogida de datos, las más relevantes de las que se abordaron en el marco del tema anterior es la encuesta. Como instrumento se utilizó un cuestionario de percepción de la labor de argumento teórico de las estrategias de la evaluación formativa.

En la validez de juicio de expertos para ambos instrumentos, se obtiene un resultado de $90 \%$, lo que supera el mínimo de 70\%, para ser considerado válido, por lo tanto, pueden ser utilizado para medir las variables en los docentes participantes.

En referencia al cuestionario sobre el argumento teórico de las estrategias de la evaluación formativa el resultado Alfa de Cronbach fue 0,952 se deduce una excelente confiabilidad y del instrumento sobre el desempeño docente en la evaluación para el aprendizaje en EBR el resultado Alfa de Cronbach fue 0,837 se expresa una excelente confiabilidad.

\section{RESULTADOS}

Nivel descriptivo 


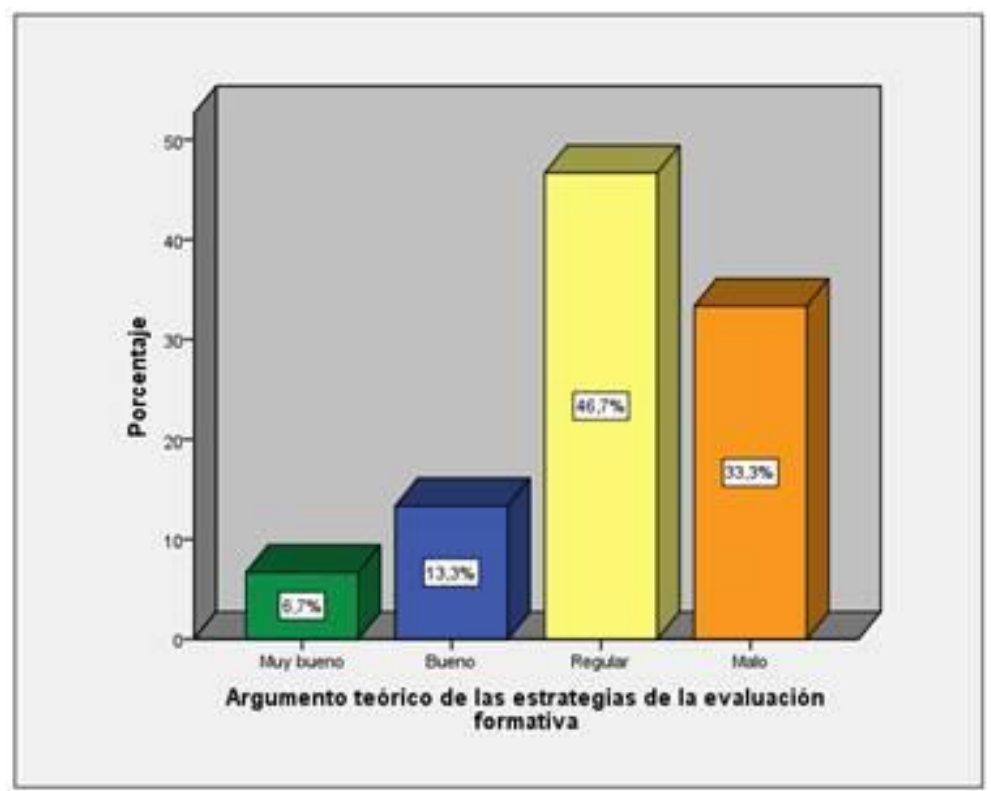

FIGURA 1.

Nivel de argumento teórico de las estrategias de la evaluación formativa

De los quince colaboradores encuestados, los resultados con referencia a este aspecto, indican que un 46,7 $\%$ (7) realizan un argumento teórico de las estrategias de la evaluación formativa regular, seguido por un 33,3 $\%$ (5) quienes realizan un argumento malo, el 13,3 \% (2) bueno y, por último, el 6,7 \% (1) muy bueno.

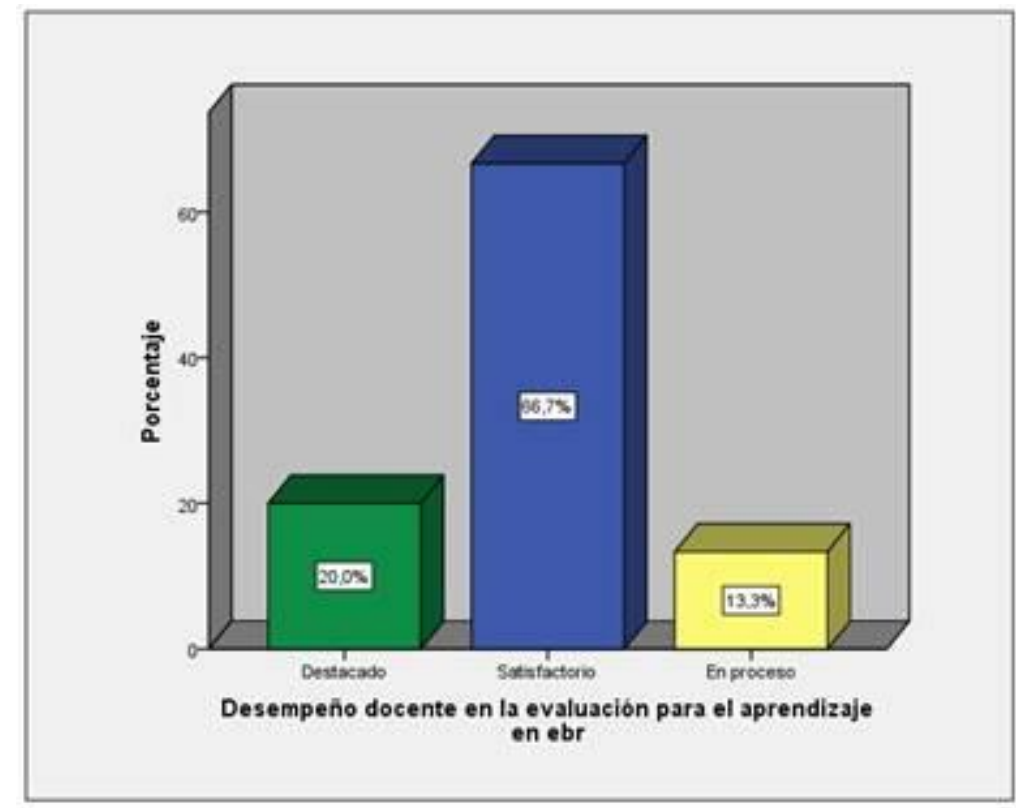

FIGURA 2.

Nivel de desempeño docente en la evaluación para el aprendizaje en EBR

De los quince colaboradores encuestados, los resultados con referencia a este aspecto, indican que un 46,7 $\%$ (7) realizan un argumento teórico de las estrategias de la evaluación formativa regular, seguido por un 33,3 $\%$ (5) quienes realizan un argumento malo, el 13,3\% (2) bueno y, por último, el 6,7 \% (1) muy bueno. 


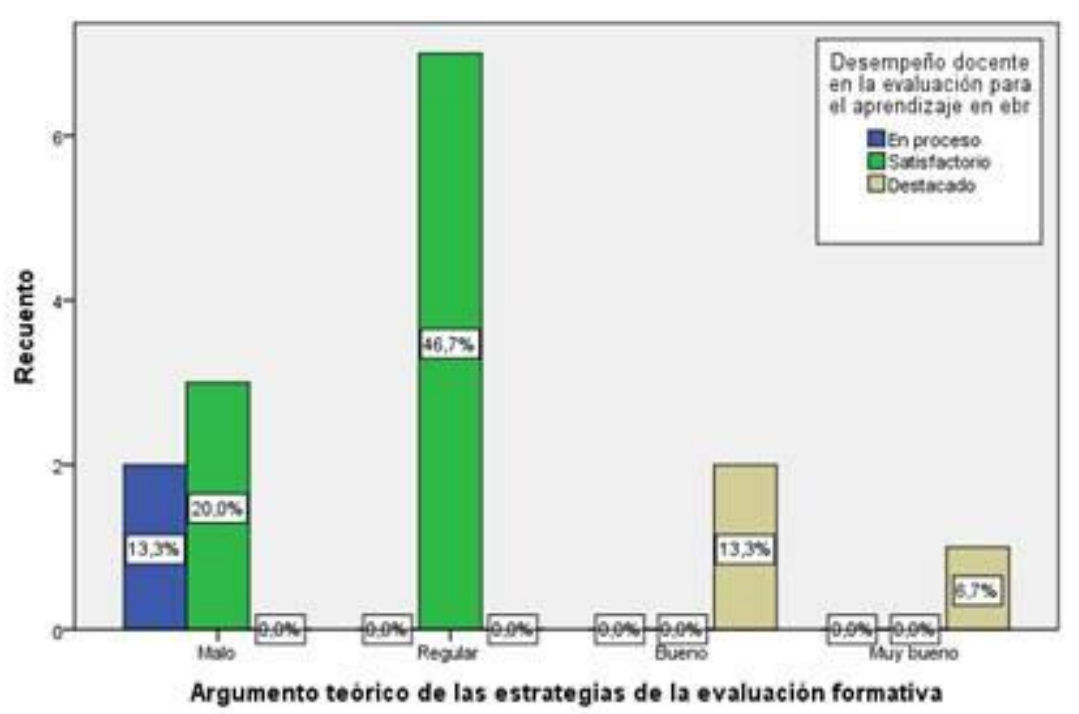

FIGURA 3.

Representación de la comparación entre los niveles hallados de acuerdo a las variables el argumento teórico de las estrategias de la evaluación formativa

y el desempeño docente en la evaluación para el aprendizaje en EBR

De los encuestados que realizan un argumento teórico de las estrategias de la evaluación formativa muy bueno, el 6,7 \% muestran un desempeño docente en la evaluación para el aprendizaje en EBR destacado; simismo, cuando realizan argumento teórico de las estrategias de la evaluación formativa bueno, el 13,3\% muestran un desempeño docente en la evaluación para el aprendizaje en EBR destacado; por otro lado, cuando realizan argumento teórico de las estrategias de la evaluación formativa regular, el 46,7 \% muestran un desempeño docente en la evaluación para el aprendizaje en EBR satisfactorio; y, por último, cuando realizan un argumento teórico de las estrategias de la evaluación formativa malo, el 20,0 \% muestran un desempeño docente en la evaluación para el aprendizaje en EBR satisfactorio y, por último, el 13,3\% en proceso.

Nivel inferencial

Prueba de normalidad.

Según Shapiro-Wilk siendo sig <0,05, aceptamos que los resultados hallados indican que las distribuciones de los datos no son normales en la muestra, en ese sentido, para contrastar las hipótesis formuladas se utilizará la estadística no paramétrica para correlaciones Rho de Spearman.

Prueba de hipótesis 
TABLA 1

Correlación y significación entre argumento teórico de las estrategias de la evaluación formativa y desempeño docente en la evaluación para el aprendizaje en EBR

\begin{tabular}{|c|c|c|c|c|}
\hline & & & $\begin{array}{l}\text { Argumento } \\
\text { teórico de } \\
\text { las } \\
\text { estrategias } \\
\text { de la } \\
\text { evaluación } \\
\text { formativa }\end{array}$ & $\begin{array}{l}\text { Desempeño } \\
\text { docente en } \\
\text { la } \\
\text { evaluación } \\
\text { para el } \\
\text { aprendizaje } \\
\text { en EBR }\end{array}$ \\
\hline \multirow{6}{*}{$\begin{array}{l}\text { Rho de } \\
\text { Spearman }\end{array}$} & \multirow{3}{*}{$\begin{array}{l}\text { Argumento } \\
\text { teórico de } \\
\text { las } \\
\text { estrategias } \\
\text { de la } \\
\text { evaluación } \\
\text { formativa }\end{array}$} & $\begin{array}{l}\text { Coeficiente } \\
\text { de } \\
\text { correlación }\end{array}$ & 1,000 &, $804^{* *}$ \\
\hline & & $\begin{array}{l}\text { Sig. } \\
\text { (bilateral) }\end{array}$ & . &, 000 \\
\hline & & $\mathrm{N}$ & 15 & 15 \\
\hline & \multirow{3}{*}{$\begin{array}{l}\text { Desempeño } \\
\text { docente en } \\
\text { la } \\
\text { evaluación } \\
\text { para el } \\
\text { aprendizaje } \\
\text { en EBR }\end{array}$} & $\begin{array}{l}\text { Coeficiente } \\
\text { de } \\
\text { correlación }\end{array}$ &, $804^{* *}$ & 1,000 \\
\hline & & $\begin{array}{l}\text { Sig. } \\
\text { (bilateral) }\end{array}$ &, 000 & . \\
\hline & & $\mathrm{N}$ & 15 & 15 \\
\hline
\end{tabular}

Se puede revisar resultados referentes a la hipótesis general, apreciando lo siguiente: en cuanto a lo obtenido en la aplicación del Rho de Spearman en el SPSS V 24, se puede decir que el rho es igual a 0,804**. Asimismo, estos datos son interpretados con una confianza del 99,99\% (**), lo cual conlleva a deducir que el margen de error es de un 0,01 bilateral, siendo esta una relación significativa positiva y además alta entre las variables de estudio, rechazando la Ho $(\mathrm{p}=0,000)$.

\section{DISCUSIÓN}

Después de realizar el análisis estadístico, se logró demostrar que la argumentación teórica de las estrategias de evaluación formativa se relaciona con el desempeño de la evaluación para el aprendizaje de los docentes de EBR que conducen la práctica preprofesional de la Facultad de Inicial de la Universidad Nacional de Educación. ( $\mathrm{p}<0,05$, Rho de Spearman $=0,804$; correlación positiva alta). Por tanto, a mayores niveles de argumento teórico de las estrategias de la evaluación formativa, mejores niveles de desempeño docente en la evaluación para el aprendizaje en EBR. En referencia a estos resultados, se pudo hallar similares hallazgos en la tesis de Villafranca (2018). Donde los resultados fueron procesados por el estadístico no probabilístico Spearman de una relación rho $=0,656$, cifra que indica una relación moderada entre los elementos de investigación, pero con un $\mathrm{p}=0,000$ indicando su significatividad, concluyendo en que las dos variables tienen una correlación significativa.

Por su parte, Quintana (2018) concluyó que los docentes poseen concepciones preestablecidas de la evaluación formativa, así como conocimientos sólidos sobre su proceso, el rol que deben realizar en el proceso de evaluación y el manejo conceptual de los instrumentos y técnicas de evaluación. Asimismo, se pudo identificar conductas didácticas ausentes, como es en el caso de la retroalimentación, regulación y el registro continuo, su ausencia expresa una inadecuada evaluación formativa en la práctica docente.

Seguidamente, se logró demostrar que la argumentación teórica de las estrategias de evaluación formativa se relaciona con la evaluación permanente del aprendizaje de los docentes de EBR que conducen la práctica 
preprofesional de la Facultad de Inicial de la Universidad Nacional de Educación. ( $\mathrm{p}<$ 0,05, Rho de Spearman $=0,572$; correlación positiva moderada). En referencia a estos resultados se pudo hallar similares hallazgos

en la tesis de Salinas (2016). Donde luego que la información obtenida fuese procesada con el coeficiente de correlación de Rho de Spearman, se obtuvo que existe una relación, aunque no de causa y efecto, sí una relación de crecimiento y/o decrecimiento paralelo.

También se halló que la argumentación teórica de las estrategias de evaluación formativa se relaciona con la toma de decisiones oportunas de los docentes de EBR que conducen la práctica preprofesional de la Facultad de Inicial de la Universidad Nacional de Educación. ( $p<0,05$, Rho de Spearman = 0,573; correlación positiva moderada). En referencia a estos resultados se pudo encontrar similares hallazgos en la tesis de Pamo (2019).

La investigación concluyó que ambas variables mantienen una cercanía de crecimiento, ya que, si uno de ellos crece, el otro también lo hace, su correlación se enmarca en una relación de comportamiento similares, a mejor valoración la otra variable también se incrementa.

Por último, se halló que la argumentación teórica de las estrategias de evaluación formativa se relaciona con la retroalimentación diferenciada de los docentes de EBR que conducen la práctica preprofesional de la Facultad de Inicial de la Universidad Nacional de Educación. ( $\mathrm{p}<0,05$, Rho de Spearman $=$ 0,607; correlación positiva alta). En referencia a estos resultados, se pudo hallar similares hallazgos en la tesis de Ortega (2015). La tesis llegó a las siguientes conclusiones: el 60 \% de docentes aplica la evaluación formativa en el aula, que se evidencia cuando los docentes buscan regular y orientar el proceso de aprendizaje de manera continua a sus estudiantes, además también comprobó que los docentes retroalimentan durante el proceso de enseñanza buscando alcanzar el logro de aprendizaje en el estudiante.

\section{CONCLUSIONES}

La argumentación teórica de las estrategias de evaluación formativa se relaciona con el desempeño de la evaluación para el aprendizaje de los docentes de EBR que conducen la práctica preprofesional de la Facultad de Inicial de la Universidad Nacional de Educación. ( $p<0,05$, Rho de Spearman = 0,804; correlación positiva alta). Por tanto, a mayores niveles de argumento teórico de las estrategias de la evaluación formativa mejores niveles de desempeño docente en la evaluación para el aprendizaje en EBR.

La argumentación teórica de las estrategias de evaluación formativa se relaciona con la evaluación permanente del aprendizaje de los docentes de EBR que conducen la práctica preprofesional de la Facultad de Inicial de la Universidad Nacional de Educación. $(\mathrm{p}<0,05$, Rho de Spearman = 0,572; correlación positiva moderada).

La argumentación teórica de las estrategias de evaluación formativa se relaciona con la toma de decisiones oportunas de los docentes de EBR que conducen la práctica preprofesional de la Facultad de Inicial de la Universidad Nacional de Educación. ( $p<0,05$, Rho de Spearman = 0,573; correlación positiva moderada).

La argumentación teórica de las estrategias de evaluación formativa se relaciona con la retroalimentación diferenciada de los docentes de EBR que conducen la práctica preprofesional de la Facultad de Inicial de la Universidad Nacional de Educación. ( $\mathrm{p}<$ 0,05, Rho de Spearman = 0,607; correlación positiva alta).

\section{RECOMENDACIONES}

Los temas de la evaluación formativa y su desempeño en la evaluación de los aprendizajes constituyen un campo de investigación en el que vale la pena invertir, por el impacto que pueden brindar en el desarrollo del aprendizaje de los estudiantes. Fue la constatación de la escasez de estudios empíricos, lo que llevó a la comunidad científica a hacer un fuerte llamado a la inversión en esta área del conocimiento. Sin embargo, existe otro motivo por el que resulta relevante y urgente profundizar en nuestros conocimientos en el ámbito de la evaluación formativa. Nuestros estudiantes necesitan la mejor orientación y apoyo para aprender y todas 
las formas que puedan iluminar este camino son bienvenidas.

La forma en que los estudiantes perciben su evaluación permanente del aprendizaje y no es independiente de que sus docentes se caractericen por tener o no prácticas de evaluación formativa. En ese sentido, se recomienda realizar las prácticas de evaluación formativa debido a que juegan un papel importante en la construcción de esta percepción.

Se recomienda la evaluación formativa para mejorar la toma de decisiones oportunas de los docentes de EBR, ya que los docentes se caracterizan por tener prácticas de evaluación formativa más consistentes que tienen expectativas más positivas en cuanto al desarrollo de su aprendizaje.

Se sugiere también la aplicación de la evaluación formativa para la retroalimentación diferenciada de los docentes de EBR, lo que más distingue a los estudiantes de los profesores con prácticas de evaluación formativa de otros, en relación con la intervención del profesor en la promoción de su aprendizaje.

\section{Referencias}

Allal, L., Cardinet, J., \& Perrenoud, P. (eds.) (1980). L'évaluation formative dans un enseignement différencié. Lang.

Black, P. \& Dylan, W. (1998). Inside the black box: raising standards through classroom assessment, Phi Delta Kappan, 80(2), 139-148. https://doi.org/10.1177\%2F003172171009200119

Hattie, J., \& Yates, G. (2018). Aprendizaje visible y el estudio de sus procesos (Trad. M. Sánchez). Ediciones Paraninfo.

Fernández, R.; Fernández, C. \& Baptista, P. (2014). Metodología de la investigación (3ª ed.). McGraw - Hill.

Ministerio de Educación. (2017). Currículo nacional de la educación básica. Lima: MINEDU.

OCDE. (2005). Evaluación formativa: mejora del aprendizaje en las aulas de secundaria. http://www.oecd.org/education/ceri/34313907.pdf

Ortega, M. (2015). Evaluación formativa aplicada por los docentes del área de Ciencia Tecnología y Ambiente en el Distrito de Hunter, Arequipa. Universidad Peruana Cayetano Heredia.

Pamo, M. (2019). Estudio contextualizado de las prácticas docentes en la evaluación formativa en el II ciclo de educación inicial de las instituciones educativas estatales de Tiabaya, 2018. (Tesis de doctorado). Universidad Nacional de San Agustín de Arequipa, Arequipa, Perú. https://bit.ly/37KjFLv

Quintana, G. (2018). La evaluación formativa de los aprendizajes en el segundo ciclo de la Educación Básica Regular en una Institución Educativa Estatal de Ate. Perú: Pontificia Universidad Católica del Perú.

Ravela, P., Leymonié, J., Viñas, J., \& Haretche, C. (2004). La evaluación en las aulas de secundaria básica en cuatro países de América Latina. Propuesta Educativa, 1 (41). 20-45. https://bit.ly/2Fyi671

Salinas, P. (2016). Relación entre argumentos teóricos de la evaluación del aprendizaje y la gestión evaluadora del docente en el aula (Tesis de maestría). Universidad San Martín de Porres, Lima, Perú. https://bit.ly/2N1dYRF

Shepard, L. (2006). La evaluación en el aula (Trad. M. Domís, 4a. ed.). México D.F.: Instituto Nacional para la Evaluación de la Educación.

Villafranca, F. (2018). Conocimiento de la evaluación formativa y la capacidad de elaboración de rúbricas de los docentes de la Red 16Ugel 02, 2018. (Tesis de maestría). Universidad César Vallejo, Lima, Perú. 